1 Fudan University School of Public Health, Shanghai, China

2 Key Laboratory of Public Health Safety, Fudan University, Ministry of Education, Shanghai, China

3 Fudan University Centre for Tropical Disease Research, Shanghai, China

4 Beijing Centre for Disease Prevention and Control and Beijing Research Centre for Preventive Medicine, Beijing, China

5 Xuhui Centre for Disease Control and Prevention, Shanghai, China

6 School of Epidemiology and Public Health, Faculty of Medicine, University of Ottawa, Ottawa, Ontario Canada

Correspondence to: Q Jiang jiangqw@fudan.edu.cn Cite this as: $B M J$ 2021;375:n2330 http://dx.doi.org/10.1136/bmi.n2330 Published: 02 December 2021
CHINA'S RESPONSE TO COVID-19

\section{Use of contact tracing, isolation, and mass testing to control transmission of covid-19 in China}

Qingwu Jiang and colleagues discuss China's experience of contact tracing, isolation, and quarantine of infected people and their contacts, and mass nucleic acid testing in the second phase of the covid-19 epidemic

Yibiao Zhou, ${ }^{1,2,3}$ Honglin Jiang, ${ }^{1,2,3}$ Quanyi Wang, ${ }^{4}$ Meixia Yang, ${ }^{5}$ Yue Chen, ${ }^{6}$ Qingwu Jiang, 2,3

China brought the first outbreak of covid-19 under control in March 2020. The response to the virus then entered a new stage aiming at preventing importation and local resurgence of covid-19. Since March 2020, only sporadic daily new cases and some small scale outbreaks in China have been seen, whereas the epidemic is still spreading globally. A total of 83249 cases were confirmed in the laboratory in mainland China when Wuhan was unblocked on 8 April 2020. Since that date, only 6315 further cases had occurred by 31 January 2021. ${ }^{1}$ The lessons learnt from the control of covid-19 in China are worth discussing.

Reinforcement of public health measures, including physical distancing, contact tracing, testing, and quarantine, are critical when an epidemic re-emerges. ${ }^{2}$ In the second phase of the epidemic in China, the response emphasised contact tracing, isolation of patients with covid-19 and their contacts, and mass testing for elimination of infections. The increase in the number of patients with the virus suggests that these measures led to substantial reductions in transmission. Nevertheless, the magnitude and impact of these outbreaks varied in different epicentres. A range of tailored approaches adapted to local conditions are required for rapid control of the pandemic. The experience of China in its search for a new sustainable normal might help other countries to design responses to covid-19.

\section{Stopping transmission}

To block the spread of covid-19, social distancing and the isolation of infected people are crucial, ${ }^{3}$ especially when vaccines are not widely available. Since the implementation throughout China of testing and centralised 14 day quarantine for all overseas arrivals, outbreaks from people entering the country are rare (fig 1). In China, an immediate response to locally emerging transmissions is to isolate people with the virus and simultaneously carry out tracing, mass testing, and quarantine of their contacts (box 1). To reduce the risks of infection, strict management measures are used to trace and isolate populations at high risk, using a broad set of criteria to define those who might have been exposed (box 2) and extending quarantine to secondary close contacts. Such a practice was shown to be necessary-for example, in a recent outbreak in Shanghai, individuals with confirmed covid-19 were found among secondary close contacts in addition to those found among primary close contacts. ${ }^{4}$ All close contacts identified through contact tracing were immediately sent to designated quarantine facilities for at least 14 days of medical observation and were tested for the virus. Timely isolation and rigorous tracking helped to interrupt transmission and reduce the magnitude of the outbreak. 

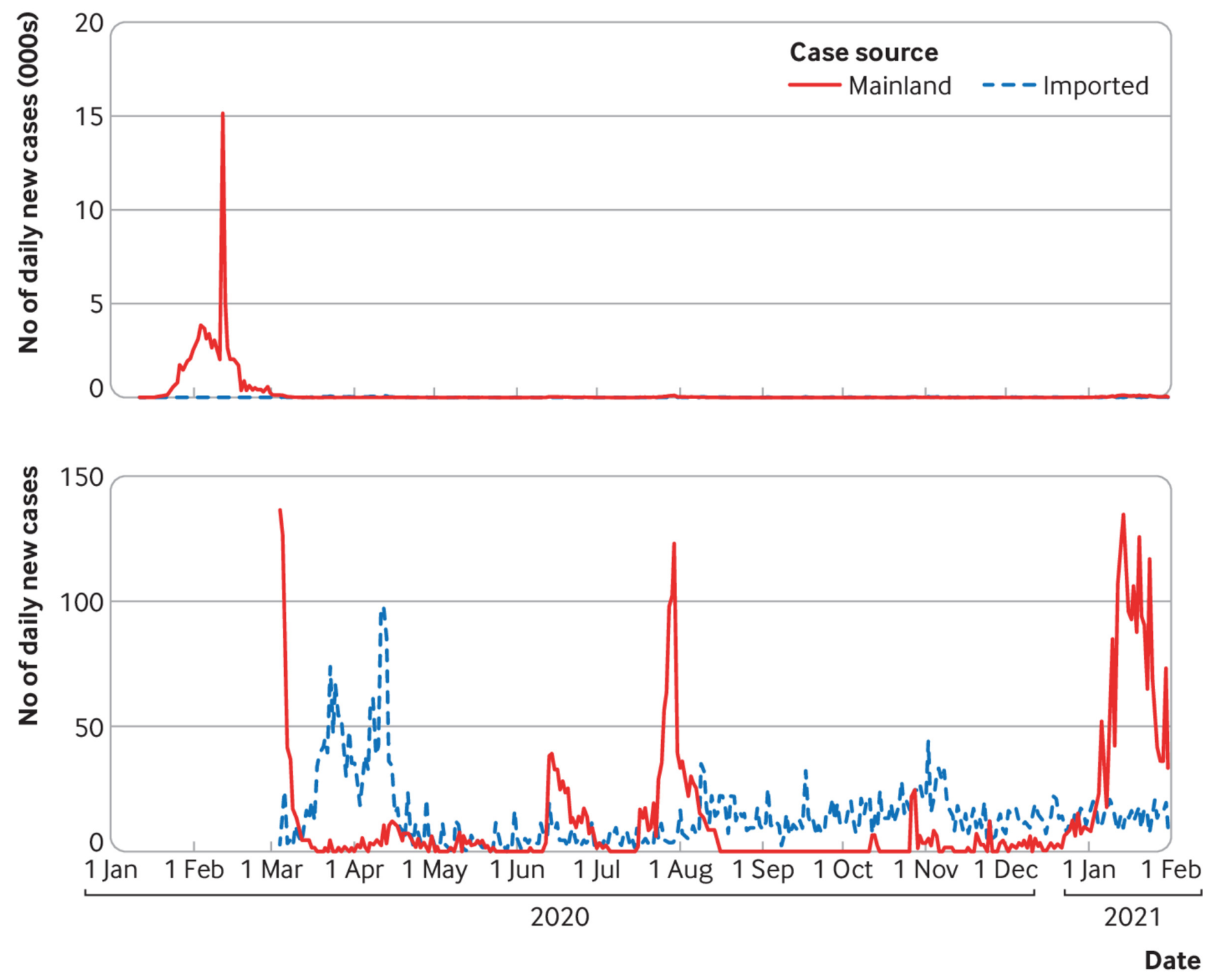

Fig 1 | The number of daily new cases of covid-19 in mainland China from January 2020 to January 2021

\section{Box 1: Public health measures to control covid-19}

- Isolation-separate a person with covid-19 infection for treatment or medical observation in designated quarantine facilities, usually a hospital or medical institution; in China, isolation is used for patients with confirmed/suspected disease and for asymptomatic carriers

- Contact tracing-identify, assess, and manage people who have been exposed to someone who has been infected with the covid-19 virus

- Quarantine of contacts-separate and restrict the movement of people who have been exposed to covid-19 virus to determine whether they have been infected; quarantine can be in a designated medical institution, hotel, or a person's own household

- Mass testing-test a group of the population for signs or symptoms related to covid-19; tests might include virological testing (eg, nucleic acid test, antigen test) and serological testing (eg, serum specific antibodies test)

\section{Box 2: Categories and definitions of exposed populations}

The exposed populations include close contacts, secondary close contacts, and general contacts. The definitions below are in compliance with the 7 th edition of the Protocol of Prevention and Control for covid-19 released by the Chinese authorities.

- Close contact-mainly refers to the primary close contact: a person who has unprotected close contact with an individual with confirmed or suspected covid-19 within two days before illness onset or with an asymptomatic carrier within two days before sampling. Criteria for a close contact include:

- Family members living in the same household

- Direct caregivers or those providing diagnosis, treatment, and nursing

- Healthcare workers providing medical services that might generate aerosols in the same space

- Any people sharing a small place, such as an office, workshop, team, elevator, cafeteria, or classroom

- Any people sharing meals or entertaining together, or providing catering and entertainment services in an enclosed environment

- Healthcare workers or family members caring for, or visiting, a patient, or any individuals having contact at a short distance 
Passengers taking the same transport having close contact (within one metre) with an infected person, including attendants and companions (eg family members, colleagues, or friends)

Any people exposed to an environment that might be contaminated by an infected person

Other people meeting criteria for close contacts as investigated and assessed by on-site investigators

- Secondary close contact-a person who has unprotected close contact (being in the same enclosed environment for living, work, a gathering, or entertainment) with a primary close contact.

- General contact-a person who has contact with a person with confirmed or suspected covid-19 or an asymptomatic carrier on public transport (eg, airplane, train, ship), or who is living, studying, working together, or seeking medical assistance, but does not meet the criteria for a close contact.
We compared two local outbreaks in China's western Xinjiang region in 2020 (fig 2). A large outbreak in Urumqi lasted for more than a month, with a total of 826 people with locally transmitted disease and five districts affected. Theoretically, people can infect others at their pre-symptomatic stage, with a high surge around two to three days before the onset of symptoms. ${ }^{5}$ The first new patient went to a hospital for medical care six days after developing symptoms, thus was not promptly detected and isolated for the period of highest infectiousness. It gave the virus more time and opportunity to spread to other close contacts quickly. Another outbreak sparked in Kashgar where the first positive patient was identified as an asymptomatic carrier and then was isolated and investigated immediately. The close contacts were subsequently found and quarantined. All infected people in this outbreak were identified during their quarantine period, indicating the need for combined isolation and tracing strategies rather than one measure alone.

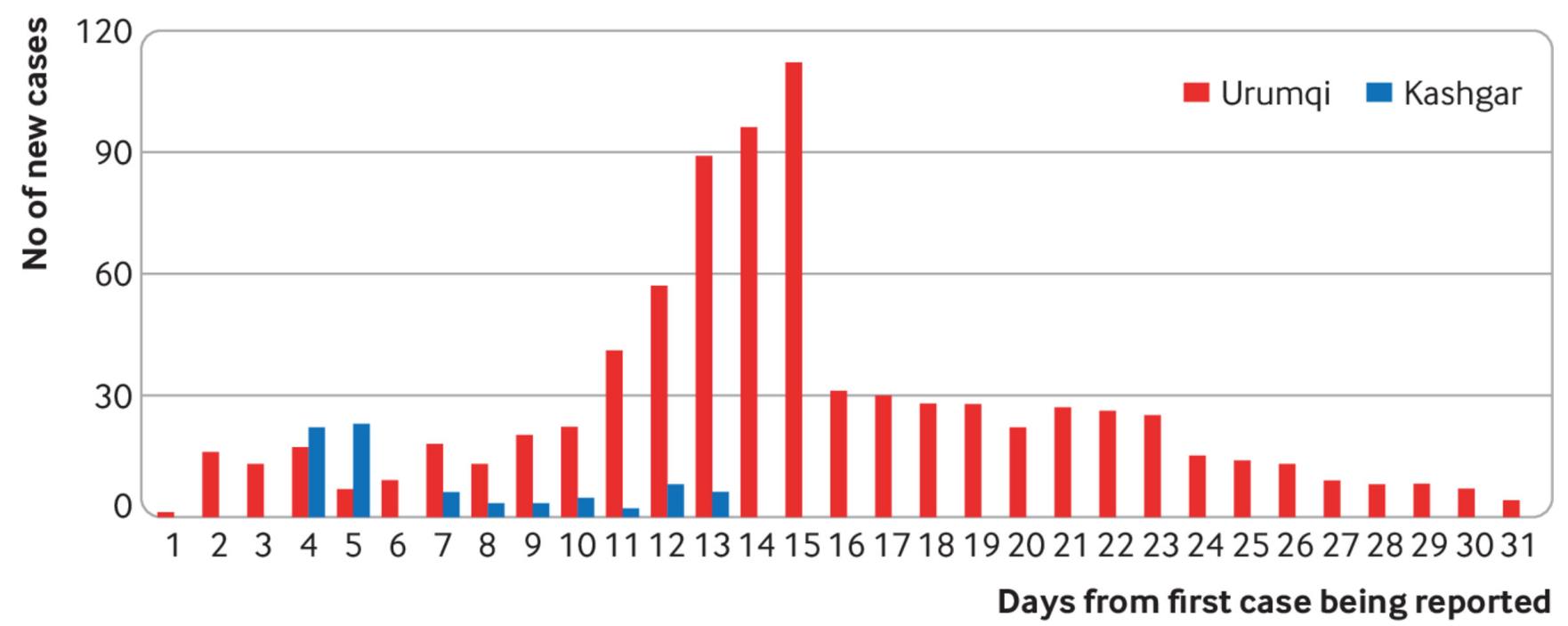

Fig 2 | Comparison of the number of people with newly confirmed covid-19 in two areas of Xinjiang region from the first day of each outbreak

\section{Identification of people with the virus}

Unique viral sequencing by nucleic acid amplification testing (NAAT) is the main approach for screening and individual diagnosis in China. This testing, performed by real time reverse transcription polymerase chain reaction, is the standard confirmation of acute SARS-CoV-2 infections, ${ }^{6}$ and can be quickly established in a new viral pandemic. Mass testing is vital to identify those infected and covid-19 hotspots and to facilitate surveillance, contact tracing, and early quarantine.

Mass testing has been applied in two situations: in response to an outbreak and for disease surveillance in populations at risk. In response to an outbreak, emergency mass testing in a targeted population is immediately activated when a new infection is reported, thus helping to detect infected people quickly and identify the source of infection. In Qingdao, China, city wide mass screening was launched to break the transmission. A total of 10.9 million people were tested within five days, and the outbreak was controlled without a lockdown. ${ }^{7}$ Similarly, New Zealand has done a large amount of testing as a measure of covid-19 control, including testing symptomatic people early in an outbreak and testing populations at high risk from specific communities. ${ }^{8}$

Nucleic acid amplification testing is also conducted in populations at risk for surveillance of the disease. According to the national prevention and control protocol, ${ }^{9}$ employees from medical institutions or key industries, such as workers at hospitals, cold-chain transport staff, couriers, taxi drivers, agricultural market vendors, and prison staff, are considered at risk of infection and should undergo regular testing. Unreported infections are projected to be $55 \%$ as contagious as documented infections per person. ${ }^{10}$ Testing of a population at risk aims to find people with active infection who are asymptomatic or pre-symptomatic so that early quarantine, tracing, and testing of close contacts, can interrupt the spread of covid-19. ${ }^{11}$

\section{Efficient epidemiological investigation for tracing and isolation}

Contact tracing based on epidemiological investigation commonly includes manual investigations (eg, phone contact, interview) and electronic tracing (eg, mobile apps, online databases). Precise epidemiological investigation in the early stage of an outbreak helps to minimise the extent of screening and the impact of the epidemic, ${ }^{4}$ but it has some limitations. Many contacts will need to be traced and tested if the incidence of symptomatic patients is high. ${ }^{12}$ In that situation, traditional investigations may be less efficient. For technological tracing to be successful, both the infected person and their contacts need to use the same software or system. Early mass testing benefits rapid detection and isolation of new instances before 
clues obtained by epidemiological investigation to prevent further transmission; thus all contacts can be found earlier and quarantined for the incubation period. By lowering the risk of further transmission, the burden of future isolation will be dramatically reduced.

A widespread community transmission struck Shijiazhuang, China at the beginning of 2021. ${ }^{13}$ One hundred and twelve people with covid-19 were confirmed within the first week in the epicentre. Sixty seven of $247(27.1 \%)$ and seven of $32(21.9 \%)$ of the positive individuals who had been missed by preliminary epidemiological investigations were found in the second and third rounds of city wide NAAT screening, respectively, indicating that this screening could help to improve the efficiency of epidemiological investigation in an emerging outbreak.

Some countries use lateral flow tests rather than NAAT for mass testing. ${ }^{14}$ Lateral flow tests detect the nucleocapsid protein antigen of SARS-CoV-2 and are a simple, rapid, and inexpensive way to test asymptomatic individuals, but less sensitive than NAAT. ${ }^{15}$ Given that the goal is to eliminate the infection in a small outbreak, lateral flow testing is not suitable for contact tracing and diagnosing covid-19. Lateral flow tests can be used as an alternative approach, however, when NAAT is not readily available, or the primary goal is to identify those people who have a high viral load and are most infectious.

\section{Pros and cons of mass testing}

Mass NAAT is of great value for reducing the duration of an epidemic and the cost of mass quarantine. If large scale testing had not been available in Wuhan, the turning point in the number of people with suspected covid-19 would have been delayed by six days, with the cost of isolation increasing by $124 \% .{ }^{16}$ Mass NAAT also contributes to containment of an outbreak in the early stage by rapidly finding infected people and facilitating epidemiological investigation for contact tracing. Furthermore, routine NAAT for a population at risk provides additional benefit by testing the asymptomatic population. It allows early detection and isolation of infectious individuals without symptoms, and consequently reduces the risk of transmission.

One of the biggest challenges faced by many countries is the cost. Mass testing requires not only procurement of covid-19 test kits but also substantial investment in equipment, laboratory facilities, and manpower. ${ }^{17}$ Many low and middle income countries do not have sufficient resources and infrastructure to test the general population. Nevertheless, some developed countries criticise mass testing owing to concern about false positive results. ${ }^{18}$ False alarms may cause unnecessary isolation and reduce the cost-benefit of large funding. To tackle these problems, it is urgent to increase testing capacity without compromising quality. More funding is needed for the development of polymerase chain reaction test kits with higher sensitivity, and digital tools to support community testing. Appropriate testing strategies should be performed in different settings. For instance, a mini-pool based strategy in which several samples are tested together, and a cheap lateral flow test can be applied for screening to reduce the cost and resource when mass NAAT is not feasible or the positive rate is low.

Other concerns include the risk of transmission caused by mass testing, public perception, and ethical issues. Measures like wearing face masks, physical distancing, education, and informed consent might be the solutions.

\section{Support for prevention and control measures in China}

To fight against the resurgence of covid-19 in many districts, China has established a well-coordinated network system for case report and registration. This network is used by national and local Centres for Disease Control and Prevention to monitor the epidemic and activate rapid response to an outbreak. The responses vary in regions based on their risk levels for an epidemic, assessed by demographic and epidemiological situations. Like other contact tracing apps, authorities rely on a health code developed by digital platforms (Alipay and WeChat) to identify people potentially exposed to covid-19. ${ }^{19}$ The health code is successfully applied in contact tracing, because these two platforms are widely used in China, and the code is mandatory when visiting public spaces (eg, hospital, shopping mall, cinema) or taking public transport. To overcome the shortage of available medical resources, three types of facilities (Fangcang shelter hospitals, refitted non-designated hospitals, and quarantine hotels) were constructed or retrofitted for centralised isolation and quarantine of people with confirmed or suspected covid-19 and close contacts. $^{20}$

Testing capacity is improving continuously in China, increasing its daily capacity for NAAT from 1.26 million copies in March 2020 to 15 million in January 2021. ${ }^{21}$ Many cities conducted several rounds of mass testing to avoid missed inspections and achieve periodic detection of infected people. To speed up mass testing, a pooled approach is applied with each pool containing five or 10 samples. Requirements and measures have been announced to strengthen mass testing. According to the state council joint prevention and control mechanism, mass NAAT of a population of five million or less should be completed in two days, and a three day limit is set for populations of more than five million people. ${ }^{22}$

\section{Conclusion}

China's experience in blocking the widespread transmission of covid-19 provides hope that rapid control might be possible. Contact tracing, isolation, and testing are the mainstay of the response to covid-19. A key lesson is that the integration and upgrading of these strategies, backed by the right technology, will boost early response to the pandemic. Optimal allocation of social resources helps to construct centralised isolation and quarantine facilities for the large numbers of people who will experience covid-19. In addition, more relevant technology, engineering, and manufacturing capacity should be redirected towards mass production of rapid testing capabilities. Notably, given inequalities between and within countries, countries and regions should not be treated homogeneously when designing response to covid-19, and appropriate, tailored management of the pandemic will inevitably be adopted.

\section{Key messages}

- Combined tracing and isolation reduces transmission of covid-19 more than either measure alone

- Mass testing, contact tracing, and early isolation in the early stage of an outbreak facilitates rapid identification of infected people

- Improvement of the capacity for mass testing and the strategy for tailored testing are important in response to future outbreaks

- A range of precise approaches should be adopted in different settings

Contributors and sources: YBZ and HLJ are joint first authors, with equal contribution to data collection analysis, and writing of the first draft. QWJ and YBZ contributed to the conceptualisation. HLJ and QYW analysed the influence and significance of mass nucleic acid testing. MXY focused on the authority's policy towards prevention and control of covid-19. YC and QWJ commented on drafts and approved the final version. QWJ is the guarantor. All data and information on the covid-19 epidemic in China mentioned in this article were collected from the websites of the National Health Commission 
(http://www.nhc.gov.cn/wjw/index.shtml) and provincial health commissions, and the English website of the state council of the People's Republic of China (http://english.www.gov.cn/).

Competing interests: We have read and understood BMJ policy on declaration of interests and have no relevant interests to declare.

Provenance and peer review: Commissioned; externally peer reviewed.

This article is part of a collection proposed by the Peking University Center for Public Health and Epidemic Preparedness and Response. Open access fees were funded by individual institutions. The $B M /$ commissioned, peer reviewed, edited, and made the decision to publish. Li-Ming Li advised on commissioning for this collection. Jin-Ling Tang, Di Wang, and Kamran Abbasi were the lead editors for The BMJ.

1 National Health Commission of the People's Republic of China. COVID-19 epidemic notification, http://www.nhc.gov.cn/xcs/yqtb/list_gzbd.shtml.

2 Cauchemez S, Kiem CT, Paireau J, Rolland P, Fontanet A. Lockdown impact on COVID-19 epidemics in regions across metropolitan France. Lancet 2020;396:1068-9.. doi: 10.1016/S0140-6736(20)32034-1 pmid: 33007219

3 Te Vrugt M, Bickmann J, Wittkowski R. Effects of social distancing and isolation on epidemic spreading modeled via dynamical density functional theory. Nat Commun 2020;11:5576. doi: 10.1038/s41467-020-19024-0 pmid: 33149128

4 Shi Y, Jiang HL, Yang MX, etal. The precision of epidemiological investigation of COVID-19 transmission in Shanghai, China. Infect Dis Poverty 2021:10:58. doi: 10.1186/s40249-021-00849-w pmid: 33947468

5 Rădulescu A, Williams C, Cavanagh K. Management strategies in a SEIR-type model of COVID 19 community spread. Sci Rep 2020;10:21256 doi: 10.1038/s41598-020-77628-4 pmid: 33277553

6 World Health Organization. Diagnostic testing for SARS-CoV-2 WHO 2020. https://www.who.int/publications/i/item/diagnostic-testing-for-sars-cov-2.

7 Xing Y, Wong GWK, Ni W, Hu X, Xing Q. Rapid response to an outbreak in Qingdao, China. N Engl J Med 2020;383:e129. doi: 10.1056/NEJMc2032361 pmid: 33207089

8 Cousins S. New Zealand eliminates COVID-19. Lancet 2020;395:1474. doi: 10.1016/S0140-6736(20)31097-7 pmid: 32386582

9 Chinese National Health Commission. [Protocol of prevention and control for COVID-19] (edition 7), 2020. http://www.nhc.gov.cn//kj/s3577/202009/318683cbfaee4191aee29cd774b19d8d.shtml

10 Li R, Pei S, Chen B, etal. Substantial undocumented infection facilitates the rapid dissemination of novel coronavirus (SARS-CoV-2). Science 2020;368:489-93. doi: 10.1126/science.abb3221 pmid: 32179701

11 Raffle AE, Pollock AM, Harding-Edgar L. Covid-19 mass testing programmes. BMJ 2020;370:m3262. doi: 10.1136/bmj.m3262 pmid: 32819920

12 Kucharski AJ, Klepac P, Conlan AJK, etalCMMID COVID-19 working group. Effectiveness of isolation, testing, contact tracing, and physical distancing on reducing transmission of SARS-CoV-2 in different settings: a mathematical modelling study. Lancet Infect Dis 2020;20:1151-60. doi: 10.1016/S1473-3099(20)30457-6 pmid: 32559451

13 Hu Y, Zhao J, Le J, etal. [Investigation of the coronavirus disease outbreak in Shijiazhuang, Hebei Province, China, at the beginning of 2021]. 2021. doi: 10.19428/j.cnki.sjpm.2021.20873.

14 Burki T. Mass testing for COVID-19. Lancet Microbe 2020;1:e317. doi: 10.1016/S2666-5247(20)30205-6. pmid: 33521731

15 García-Fiñana M, Hughes DM, Cheyne CP, etal. Performance of the Innova SARS-CoV-2 antigen rapid lateral flow test in the Liverpool asymptomatic testing pilot: population based cohort study. BMJ 2021;374:n1637. doi: 10.1136/bmj.n1637 pmid: 34230058

16 Xie Q, Wang J, You JL, etal. [The role of large-scale testing platform in the prevention and control of the COVID-19 pandemic: an empirical study based on a novel numerical model]. Zhonghua $Y$ Xue Za Zhi2020;100:2532-6. doi: 10.3760/cma.j.cn112137-20200320-00860. pmid: 32829601

17 Lau LL, Hung N, Wilson K. COVID-19 response strategies: considering inequalities between and within countries. Int J Equity Health 2020;19:137. doi: 10.1186/s12939-020-01254-9 pmid: 32787914

18 lacobucci G. Covid-19: Government faces criticism over $£ 500 \mathrm{~m}$ plan to pilot mass testing. BMJ 2020;370:m3482. doi: 10.1136/bmi.m3482. pmid: 32895227

19 Liang F. Covid-19 and health code: how digital platforms tackle the pandemic in China. Soc Media Soc 2020;6:2056305120947657. doi: 10.1177/2056305120947657 pmid: 34192023

20 Wang $X$, Wang J, Shen J, etal. Facilities for centralized isolation and quarantine for the observation and treatment of patients with COVID-19: experience from Wuhan, China. Engineering (Beijing) 2021. doi: 10.1016/j.eng.2021.03.010. pmid: 33903828

21 State Council of the People's Republic of China. China's daily COVID-19 testing capacity hits 15 million samples. 2021. http://english.www.gov.cn/statecouncil/ministries/202101/27/content_WS6010fbcdc6d0f7257694498f.htm

22 State Council of the People's Republic of China. China strengthens nucleic acid testing. 2021 http://english.www.gov.cn/news/topnews/202108/11/content_WS6113020ec6d0df57f98de478.htm

This is an Open Access article distributed in accordance with the Creative Commons Attribution Non Commercial (CC BY-NC 4.0) license, which permits others to distribute, remix, adapt, build upon this work non-commercially, and license their derivative works on different terms, provided the original

work is properly cited and the use is non-commercial. See: http://creativecommons.org/licenses/by$\mathrm{nc} / 4.0 /$. 